\title{
The Contradiction between Two Versions of Quantum Theory Could Be Decided by Experiment
}

\author{
María Esther Burgos \\ Faculty of Sciences, University of Los Andes, Mérida, Venezuela \\ Email: mburgos25@gmail.com
}

How to cite this paper: Burgos, M.E. (2019) The Contradiction between Two Versions of Quantum Theory Could Be Decided by Experiment. Journal of Modern Physics, 10, 1190-1208. https://doi.org/10.4236/jmp.2019.1010079

Received: June 10, 2019

Accepted: September 9, 2019

Published: September 12, 2019

Copyright $\odot 2019$ by author(s) and Scientific Research Publishing Inc. This work is licensed under the Creative Commons Attribution International License (CC BY 4.0).

http://creativecommons.org/licenses/by/4.0/

\section{(c) (i) Open Access}

\begin{abstract}
The incompatibility of Orthodox Quantum Mechanics with philosophical realism poses a serious challenge to scientists upholding such a philosophical doctrine. The desire to find a solution to this and other conceptual problems that quantum mechanics confronts has motivated many authors to propose alternative versions to Orthodox Quantum Mechanics. One of them is the Spontaneous Projection Approach, a theory grounded on philosophical realism. It has been introduced in previous papers and, with a few exceptions, it yields experimental predictions coincident with those of Orthodox Quantum Mechanics. One of these exceptions is analyzed in detail. The difference in predictions becomes apparent in a suggested experiment which could put both theories to the test.
\end{abstract}

\section{Keywords}

Philosophical Realism, Orthodox Quantum Mechanics, Spontaneous

Projection Approach, Quantum Measurement Problem

\section{Introduction and Outlook}

Realism is a philosophical doctrine that revolves around two theses: the first (or ontological thesis) is that the world exists by itself as opposed to being the product of human mind; the second (or epistemological thesis) is that it can be known gradually and approximately [1]. Among authors adopting realism let us mention Albert Einstein and Mario Bunge. For Einstein, "The belief in an external world independent of the perceiving subject is the basis of all natural science" [2]. For Bunge, "Epistemological realism is the family of epistemologies which assume that a) the world exists independently of the knowing subject, and 
b) the task of science is to produce maximally true conceptual models of reality..." ([3] pp. 191-192).

In 1930, Paul Dirac published the first formulation of quantum mechanics [4]. Two years later John von Neumann published Mathematische Grundlagen der Quantenmechanik [5]. These first versions of quantum theory share two characteristics: 1) the state vector $|\psi\rangle$ (wave function $\psi$ ) describes the state of an individual system, and 2) they involve two laws of change of the state of the system: spontaneous (natural) processes, governed by the Schrödinger equation; and measurement processes, ruled by the projection postulate. Many other versions of quantum theory followed. Those where $|\psi\rangle$ describes the state of an individual system and the projection postulate is included among its axioms are generally called standard, ordinary or Orthodox Quantum Mechanics (OQM), sometimes referred to as the Copenhagen Interpretation.

On the one hand, OQM is very successful a theory. "[It has provided] a strikingly successful recipe for doing calculations that accurately described the outcomes of experiments... [It has been] instrumental in predicting antimatter, understanding radioactivity (leading to nuclear power), accounting for the behavior of materials such as semiconductors, explaining superconductivity and describing interactions such as those between light and matter (leading to the invention of the laser) and of radio waves and nuclei (leading to magnetic resonance imaging). Many successes of quantum mechanics involve its extension, quantum field theory, which forms the foundations of elementary particle physics..." [6].

On the other hand, OQM contravenes philosophical realism. This is particularly clear as concerns its projection postulate. Scientists upholding philosophical realism have criticized this postulate because it introduces a subjective element into the theory: either it invokes an observer placed above the laws of nature ([3], pp. 191-202) or it appeals to the interaction between the quantum system and a macroscopic measuring device (built, of course, by human beings). Referring to this issue Max Jammer points out: "As long as quantum mechanics one-body or many-body system does not interact with macroscopic bodies, as long as its motion is described by the deterministic Schrödinger time-dependent equation, no events could be considered to take place in the system... If the whole physical universe were composed only of microphysical entities, as it should be according to the atomic theory, it would be a universe of evolving potentialities (timedependent $\psi$ functions) but not of real events" ([7], p. 474).

It is worth noting that well-known scientists dealing with quantum mechanics renounce philosophical realism, either implicitly or explicitly. Let us mention two of them: Niels Bohr and Asher Peres. The last one declares: "Quantum theory is not an objective description of physical reality" ([8], p. 423) and "any attempt to inject realism in physical theory is bound to lead to inconsistencies" [9]. In his analysis of Bohr's philosophy Aage Petersen asserts: "When asked whether the algorithm of quantum mechanics could be considered as somehow mirroring an underlying quantum world, Bohr would answer: 'There is no 
quantum world. There is only an abstract quantum physical description. It is wrong to think that the task of physics is to find out how nature is. Physics concerns what we can say about nature'." [10].

Taking philosophical realism as a starting point, a Spontaneous Projection Approach (SPA) to quantum theory was formulated some years ago [11]. This approach was recently modified to account for quantum processes in the general case, including those where the Hamiltonian depends explicitly on time [12]. It is worth noting that SPA overcomes the main flaws of OQM [12] and in general yields experimental predictions coincident with those of OQM. There are, however, a few exceptions which could be exploited to confront both theories. This is the principal aim of our present study.

The contents of this paper are as follows: In Section 2, we reproduce the formulations of OQM and SPA, highlight their similarities and differences, mention some contributions aiming to solve the measurement problem and refer one of them in detail. In Section 3, we deal with a case where SPA and OQM yield different experimental predictions. We consider a silver atom in the ground state placed in a uniform constant magnetic field $\boldsymbol{B}$. Starting with the atom in a given state we find 1) the evolution of its state assuming the validity of OQM; and 2) the possible changes of its state assuming the validity of SPA. The resulting contradiction could be decided by means of a suggested experiment. Section 4 is devoted to the discussion and conclusions.

\section{OQM, SPA and the Measurement Problem}

\subsection{Formulation of $\mathrm{OQM}$}

The formulation of quantum mechanics due to von Neumann (OQM) ([7], p. 5) ([13], pp. 215-222) includes the primitive (undefined) notions of system, state and physical quantity (or observable). Its postulates are:

I) To every system $\zeta$ corresponds a Hilbert space $H b$ whose vectors (state vectors, wave functions) $|\psi\rangle$ completely describe the states of the system.

II) To every physical quantity $\mathcal{A}$ corresponds uniquely a self-adjoint operator $A$ acting in $H b$. It has associated the eigenvalue equations

$$
A\left|\alpha_{k}^{v}\right\rangle=\alpha_{k}\left|\alpha_{k}^{v}\right\rangle
$$

( $v$ is introduced in order to distinguish between the different eigenvectors that may correspond to one eigenvalue $\alpha_{k}$ ), and the closure relation

$$
\sum_{k, v}\left|\alpha_{k}^{v}\right\rangle\left\langle\alpha_{k}^{v}\right|=\mathcal{J}
$$

is fulfilled (here $\mathcal{J}$ is the identity operator). If either $k$ or $v$ is continuous, the respective sum has to be replaced by an integral.

III) Spontaneous processes are continuous. The evolution in time $t$ of the state vector $|\psi(t)\rangle$ is governed by the Schrödinger equation

$$
i \hbar \frac{\mathrm{d}}{\mathrm{d} t}|\psi(t)\rangle=H(t)|\psi(t)\rangle
$$


where $H(t)$ is the Hamiltonian of the system, $\hbar$ Planck's constant divided by $2 \pi$ and $i$ the imaginary unit.

Comment: The Schrödinger equation is a deterministic law. The solution $|\psi(t)\rangle$ of Equation (3) which corresponds to the initial condition $|\psi(0)\rangle$ is unique. The system's state evolves according to the equation

$$
|\psi(t)\rangle=U(t, 0)|\psi(0)\rangle
$$

where $U(t, 0)$ is the evolution operator corresponding to the Hamiltonian $H(t)$; more details in ([4], p. 109) ([13], p. 308) ([14], p. 137).

IV) For a system in the state $|\psi\rangle$ the probability that the result of a measurement of $\mathcal{A}$ lies between $\alpha_{1}$ and $\alpha_{2}$ is $\|\eta\|^{2}$, where $\|\eta\|$ is the norm of $|\eta\rangle=\left(\mathcal{J}_{\alpha_{2}}-\mathcal{J}_{\alpha_{1}}\right)|\psi\rangle$ and $\mathcal{J}_{\alpha}$ is the resolution of the identity belonging to $A$.

V) Projection postulate. If a measurement of $\mathcal{A}$ yields a result between $\alpha_{1}$ and $\alpha_{2}$, then the state of the system immediately after the measurement is an eigenfunction of $\left(\mathcal{J}_{\alpha_{2}}-\mathcal{J}_{\alpha_{1}}\right)$.

\subsection{Formulation of SPA}

The formulation of SPA includes the primitive notions of system, state, physical quantity and probability [12]. Its postulates are:

A) Agrees word for word with postulate I of OQM.

B) Agrees word for word with postulate II of OQM.

C) Spontaneous processes can be either continuous or discontinuous. In continuous processes the evolution in time of the state vector $|\psi\rangle$ is determined by the Schrödinger equation.

Comment (i): Equation (4) applies to processes that are continuous in the whole time interval $(0, t)$. In cases where this equation is satisfied, we shall say that in the interval $(0, t)$ the system remains in the Schrödinger channel.

Comment (ii): Let the Hamiltonian of the system be

$$
H(t)=\varepsilon+\lambda(t)
$$

where $\varepsilon$ is the sum of all the terms of the Hamiltonian which do not depend explicitly on time $t$ and $\lambda(t)$ includes every term of the Hamiltonian depending explicitly on $t$. If $\lambda(t)=0$, a self-adjoint operator $A$ fulfilling

$$
\begin{gathered}
\frac{\partial A}{\partial t}=0 \\
{[A, \varepsilon]=0}
\end{gathered}
$$

is a constant of the motion.

Definition of preferential states: The system in the state $|\psi(t)\rangle$ has tendency to jump to the eigenstates of operators satisfying Equations (6) and (7) whether $\lambda(t)=0$ or not [12]. If there is a unique set of $N \geq 2$ orthonormal vectors: $\left|\varphi_{1}\right\rangle,\left|\varphi_{2}\right\rangle, \cdots,\left|\varphi_{N}\right\rangle \quad\left(\left\{N_{\varphi}\right\}\right.$ for short $)$ such that the normalized state of the system $\zeta$ at time $t$ can be written 


$$
|\psi(t)\rangle=\sum_{j} \gamma_{j}(t)\left|\varphi_{j}\right\rangle
$$

where 1) $\gamma_{j}(t)=\left\langle\varphi_{j} \mid \psi(t)\right\rangle \neq 0$ for every $\left.j=1,2, \cdots, N ; 2\right)$ at least $(N-1)$ vectors belonging to the set $\left\{N_{\varphi}\right\}$ are eigenstates of $\varepsilon$; and 3) every selfadjoint operator $A$ for which Equations (6) and (7) are valid satisfies the relation

$$
\langle\psi(t)|A| \psi(t)\rangle=\sum_{j}\left|\gamma_{j}(t)\right|^{2}\left\langle\varphi_{j}|A| \varphi_{j}\right\rangle
$$

we shall say that $\left\{N_{\varphi}\right\}$ is the preferential set of $\zeta$ in the state $|\psi(t)\rangle$ and the members of $\left\{N_{\varphi}\right\}$ will be called its preferential states.

Comment (iii): The validity of Equation (9) ensures the statistical sense of conservation laws [11]. Note that, by definition, the preferential set does not depend on $\lambda(t)$ [12].

D) If the system $\zeta$ in the state $|\psi(t)\rangle$ has no preferential set, it remains in the Schrödinger channel.

E) If the system $\zeta$ in the normalized state $|\psi(t)\rangle$ has the preferential set $\left\{N_{\varphi}\right\}$, in the small time interval $(t, t+\mathrm{d} t)$ it can either remain in the Schrödinger channel or jump to one of its preferential states $\left|\varphi_{k}\right\rangle$ with probability

$$
\mathrm{d} P_{k}(t)=\left|\gamma_{k}(t)\right|^{2} \frac{\mathrm{d} t}{\tau(t)}
$$

where

$$
\tau(t) \Delta \varepsilon(t)=\frac{\hbar}{2}
$$

and

$$
[\Delta \varepsilon(t)]^{2}=\left\langle\psi(t)\left|\varepsilon^{2}\right| \psi(t)\right\rangle-[\langle\psi(t)|\varepsilon| \psi(t)\rangle]^{2}
$$

Comment: The probability that the system will remain in the Schrödinger channel during the time interval $(t, t+\mathrm{d} t)$ is

$$
\mathrm{d} P_{S}(t)=1-\frac{\mathrm{d} t}{\tau(t)}
$$

see [12]. If the parameter $\tau$ defined by Equation (11) is constant, the state $|\psi(t)\rangle$ may be considered as an unstable state that can decay to one of its $N$ preferential states [11] [15] [16]. In this case the probability that the system will remain in the Schrödinger channel during the interval $(0, t)$ is a decreasing exponential

$$
P_{S}(t)=\mathrm{e}^{-t / \tau}
$$

with relaxation time $\tau$.

\subsection{OQM, SPA and Other Contributions Aiming to Solve the Measurement Problem}

OQM assumes that there are two kinds of processes: spontaneous processes and 
measurement processes. In spontaneous processes the evolution of the state vector is governed by the Schrödinger equation; in measurement processes the changes of the state vector are ruled by the projection postulate.

On the one hand, since the Schrödinger equation involves a derivative of the state vector $|\psi(t)\rangle$ with respect to time $t$, in spontaneous processes $|\psi(t)\rangle$ must be continuous. On the other hand, Postulate C of SPA states that in continuous processes the evolution in time of the state vector $|\psi(t)\rangle$ is determined by the Schrödinger equation. Continuous processes are governed by the Schrödinger equation in $S P A$ as well as in $O Q M$.

It is worth stressing that continuous processes are not restricted to cases where the system in the state $|\psi(t)\rangle$ has no preferential set. Even if the system in the state $|\psi(t)\rangle$ has a preferential set, the dominant process in a small time interval $(t, t+\mathrm{d} t)$ is the Schrödinger evolution; see Equation (13). According to Equation (14) the Schrödinger evolution is also the dominant process for $t \ll \tau$. Spontaneous projections seldom occur.

OQM measurements are somehow related to SPA projections. Let us highlight their similarities: SPA projections as well as OQM measurements 1) yield discontinuities of the state vector; 2 ) instantaneously break down certain superposition of different states; 3 ) violate conservations laws but respect their statistical sense [16] [17]; 4) imply a kind of action-at-a-distance [16] [18]; and 5) collide with determinism [16] [19].

The main difference between SPA and OQM is that while according to SPA the state vector $|\psi(t)\rangle$ may spontaneously jump to one of its preferential states, e.g. $\left|\varphi_{n}\right\rangle$ (see Equation (8)), OQM ensures that $|\psi(t)\rangle$ remains continuous until a measurement is performed.

Let $A$ be the operator representing the physical quantity $\mathcal{A}$ to be measured and $\left|\alpha_{j}\right\rangle$ the eigenvector of $A$ corresponding to the eigenvalue $\alpha_{j}$ (for simplicity we refer to the discrete non-degenerate case). The state vector $|\psi(t)\rangle$ can be expanded

$$
|\psi(t)\rangle=\sum_{j} c_{j}(t)\left|\alpha_{j}\right\rangle
$$

where $c_{j}(t)=\left\langle\alpha_{j} \mid \psi(t)\right\rangle$. OQM states that the probability that the measurement of $\mathcal{A}$ will yield the result $\alpha_{n}$ and so (according to the projection postulate) $|\psi(t)\rangle$ will jump to $\left|\alpha_{n}\right\rangle$ is

$$
p_{n}(t)=\left|c_{n}(t)\right|^{2}
$$

According to SPA the probability that in the small time interval $(t, t+\mathrm{d} t)$ the state vector $|\psi(t)\rangle$ will jump to the preferential state $\left|\varphi_{n}\right\rangle$ is

$$
\mathrm{d} P_{n}(t)=\left|\gamma_{n}(t)\right|^{2} \frac{\mathrm{d} t}{\tau(t)}
$$

see Equation (10). If the relaxation time $\tau$ and $\left|\gamma_{n}(t)\right|^{2}=\left|\gamma_{n}\right|^{2}$ are constant in the interval $(0, t)$, the probability that $|\psi(t)\rangle$ will jump to $\left|\varphi_{n}\right\rangle$ in this interval 
is

$$
P_{n}(t)=\left|\gamma_{n}\right|^{2}\left(1-\mathrm{e}^{-t / \tau}\right)
$$

[11]. For $t \gg \tau$, it results

$$
P_{n}(t \gg \tau) \simeq\left|\gamma_{n}\right|^{2}
$$

The similarity of the mathematical expressions for the probabilities given by (16) and (19) is worth stressing.

Even if it is not clear what a measurement is [20], the notion of measurement is included in two of the five postulates of OQM formulation. In any case, collapses or something similar to collapses are necessary to avoid paradoxes such as that of Schrödinger's cat ([7], pp. 216-217). "Because of the linearity of the Schrödinger evolution there is no mechanism to stop the evolution and yield a single result for the measurement" ([14], p. 264). However, "in common life as well as in laboratories, one never observe superposition of results; we observe that Nature seems to operate in such a way that a single result always emerges from a single experiment; this will never be explained by the Schrödinger equation, since all that it can do is to endlessly extend its ramifications into the environment, without ever selecting one of them only" [21]. The rule compelling $|\psi(t)\rangle$ to remain continuous as long as no measurements are performed poses a serious problem for OQM. By contrast, assuming that spontaneous projections are natural processes, SPA succeeds in stopping the endless ramifications resulting from Schrödinger evolution.

The issues just mentioned are at the very heart of the measurement problem. In previous articles we dealt with several proposed solutions to this problem. Among them, 1) Margenau's contribution ([7], pp. 226-227) was addressed in [16]; 2) The statistical interpretation of quantum mechanics [22] [23] was addressed in [16];3) Bohm's theory [24] [25] was addressed in [11]; 4) Many-worlds interpretation [26] [27] was addressed in [16]; 5) The consistent histories approach to quantum mechanics [28] [29] was addressed in [11]; 6) Decoherence [30] [31] was addressed in [16]; 7) The theory of spontaneous localizations [32] [33] was addressed in [11]; 8) Bell's Beables for quantum field theory [34] was addressed in [15]; 9) Sudbery's privileged observables [35] was addressed in [15]; and 10) Sudbery's preferred observables [36] was addressed in [15]. Some of them as well as SPA involve dynamical reduction models.

\subsection{SPA Preferential Sets and Sudbery's Preferred Observables}

Among the contributions appearing in the previous list the closest to SPA is Sudbery's theory of preferred observables. Let us highlight their similarities and differences with SPA.

In his article entitled Diesse Verdammten Quantenspringerei, Anthony Sudbery asserts: "there are no events in quantum theory. The nearest thing to an event described in the basic theory is the result of a measurement... There is no way that the theory can describe events, like the $\Omega^{-}$decay... which happen spon- 
taneously and are passively recorded by the waiting experimenter... Nevertheless, textbooks do purport to derive decay rates for such events from the general principles of quantum mechanics. The derivation goes like this. To describe a decay $A \rightarrow B+C$, we start at time $t=0$ with a state $|A\rangle$ in which the unstable particle $A$ is certain not to have decayed, and follows its time evolution, governed by the Hamiltonian $H$, to a superposition of the initial state and a state of the decay products $B$ and $C$ :

$$
\mathrm{e}^{-i H t / \hbar}|A\rangle=a(t)|A\rangle+b(t)|B C\rangle
$$

The general principles of quantum mechanics are then supposed to yield the interpretation that $|b(t)|^{2}$ is the probability that by time $t$ there has been a transition from particle $A$ to particles $B+C$. But where does this notion of a 'transition' come from? It appears nowhere in the general principles of the theory as usually stated. The literal application of these principles to the state (20) gives only the statement that if a measurement is made at time $t$ (to determine, say, whether a particle of type $B$ is present) then $|b(t)|^{2}$ is the probability that the result of the measurement will be positive. The inference that something discontinuous (a transition) happened between time 0 and time $t$ is completely unwarranted. According to the official principles, quantum systems evolve continuously (as the time dependence of (20) shows), and quantum jumps occur only when provoked by the intervention of an experimenter" [36].

Sudbery adds: "The procedure that starts with the time-depending state vector (20), produces from it a time-dependent probability $P(t)$, interprets this as a probability of something having happened, and, on the strength of this interpretation, derives from $P(t)$ a transition probability per unit time, certainly ends up with an empirical adequate result" [36]. The problem Sudbery wants to discuss is that of formulating the theory so as to make this argument as sound as its conclusion. To do so, he claims, "transitions must be given a fundamental role in the theory; one of its basic postulates should be of the form 'If the system [however broadly conceived it] is in the state $\psi$ at time $t$, there is a probability $T_{\varphi \psi}(t) \mathrm{d} t$ that it will take a transition to state $\varphi$ between $t$ and $t+\mathrm{d} t$.' Such a postulate, if it is to be fundamental, would need to be accompanied by a clear statement of exactly what the eligible states $\varphi, \psi$ are" [36].

In order to formulate a postulate concerning transitions, Sudbery adopts a suggestion made by Bell [34]. "The basic idea is that there is a set of special physical quantities, which have a fundamental status; Bell liked to call them beables... These quantities always have definite values which change stochastically according to transition probabilities determined by the solutions to the Schrödinger equation. Equivalently, one can replace the special quantities by a special set of subspaces of space states, namely their eigenspaces, and the actual values of special quantities by a vector (the projection of the full space vector) in the corresponding subspace" [36]. These subspaces are called the viable subspaces. 
By adopting Bell's transition probabilities, Sudbery succeeds in giving a satisfactory description of the decay process. But, he points out, "Bell's formulation of quantum mechanics does not exists until one has specified the viable subspaces. In this respect, it is no improvement on the conventional formulation, which does not exist until one has specified precisely which physical arrangement constitutes 'measurement'... If one is aspiring to give an absolute description of the physical world, there seems to be no good empirical or theoretical reason why any particular set of physical quantities should have fundamental status" [36]. Nevertheless, he adds, "if arbitrary choices cannot be avoided, we must consider how to live with them" [36].

SPA fulfills all the requirements stated by Sudbery: According to Postulate E, if the system is in the state $|\psi(t)\rangle$ and $\left|\varphi_{k}\right\rangle$ is one of its preferential states, the probability that it will take a transition from $|\psi(t)\rangle$ to $\left|\varphi_{k}\right\rangle$ between $t$ and $t+\mathrm{d} t$ is

$$
\mathrm{d} P_{k}(t)=\left|\gamma_{k}(t)\right|^{2} \frac{\mathrm{d} t}{\tau(t)}
$$

where $\gamma_{k}(t)=\left\langle\varphi_{k} \mid \psi(t)\right\rangle$. SPA explicitly says what states $|\psi(t)\rangle$ and $\left|\varphi_{k}\right\rangle$ are; neither special physical quantities nor viable subspace are necessary for SPA to exist. Physical quantities represented by operators satisfying Equations (6) and (7) have a fundamental status; but as opposed to Bell's beables neither their choices are arbitrary nor they have definite values at all times.

\section{A Case Where SPA and OQM Yield Different Experimental Predictions}

In this section we shall deal with a case where SPA and OQM yield different experimental predictions. Let us consider a silver atom in the ground state placed in a uniform constant magnetic field $\boldsymbol{B}$. We choose $O z$ axis along $\boldsymbol{B}$. Let $S_{z}$ be the operator representing the $z$-component of the atom's spin $S$. The eigenvectors of $S_{z}$ corresponding to the eigenvalues $+\frac{\hbar}{2}$ and $-\frac{\hbar}{2}$ will be denoted $\left|+_{z}\right\rangle$ and $\left|-_{z}\right\rangle$ respectively. They satisfy the equations

$$
\begin{gathered}
S_{z}\left|+_{z}\right\rangle=+\frac{\hbar}{2}\left|+_{z}\right\rangle \\
S_{z}\left|-_{z}\right\rangle=-\frac{\hbar}{2}\left|-_{z}\right\rangle \\
\left\langle+_{z} \mid-_{z}\right\rangle=\left\langle-_{z} \mid+_{z}\right\rangle=0 \\
\left\langle+_{z} \mid+_{z}\right\rangle=\left\langle-_{z} \mid-_{z}\right\rangle=1 \\
\left|+_{z}\right\rangle\left\langle+_{z}|+|-_{z}\right\rangle\left\langle--_{z}\right|=\mathcal{J}
\end{gathered}
$$

Let $\boldsymbol{u}$ be a unitary vector defined by the angles $\vartheta$ and $\varphi$ (Figure 1). The operator representing the $u$-component of $\boldsymbol{S}$ will be denoted $S_{u}$. Its eigenvector corresponding to the eigenvalue $+\frac{\hbar}{2}$ satisfies the equation 


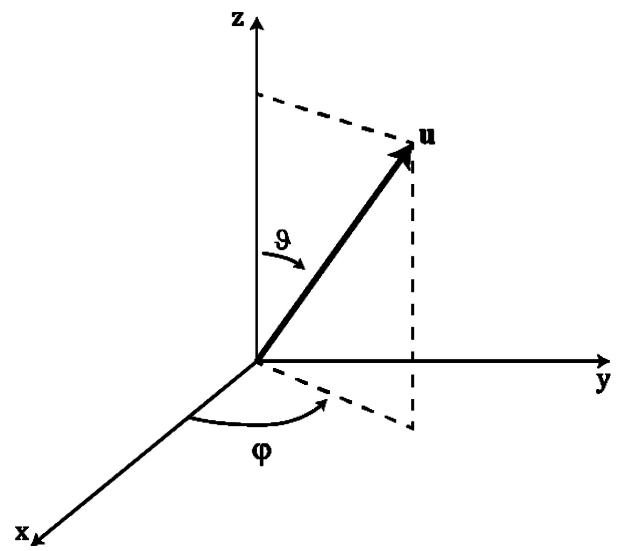

Figure 1 . The unitary vector $\boldsymbol{u}$ is defined by the angles $\vartheta$ and $\varphi$.

$$
S_{u}\left|{ }_{u}\right\rangle=+\frac{\hbar}{2}\left|{ }_{u}\right\rangle
$$

and its expansion on the basis of the eigenvectors of $S_{z}$ is

$$
\left|+_{u}\right\rangle=\cos \frac{\vartheta}{2} \mathrm{e}^{-i \varphi / 2}\left|+_{z}\right\rangle+\sin \frac{\vartheta}{2} \mathrm{e}^{i \varphi / 2}\left|-_{z}\right\rangle
$$

See for instance ([13], p. 395).

We shall refer only to spin states. The Hamiltonian of a silver atom in the ground state placed in a uniform magnetic field is time-independent and can be written

$$
\varepsilon=\omega S_{z}
$$

where

$$
\omega=-g B
$$

is the Larmor frequency, $g$ the gyromagnetic ratio of the silver atom in the ground state and $B$ the modulus of the magnetic field ([13], p. 403). Taking into account Equation (29), the eigenvalue equations of this Hamiltonian can be written

$$
\begin{aligned}
& \varepsilon\left|+_{z}\right\rangle=E_{+}\left|+_{z}\right\rangle=+\frac{\hbar \omega}{2}\left|+_{z}\right\rangle \\
& \varepsilon\left|-_{z}\right\rangle=E_{-}\left|-_{z}\right\rangle=-\frac{\hbar \omega}{2}\left|-_{z}\right\rangle
\end{aligned}
$$

where $E_{+}$and $E_{-}$are the eigenvalues of $\varepsilon$.

Magnetic fields are never completely uniform and the wave functions of atoms always have a certain extension. The Hamiltonian given by Equation (29) is a valid approximation only in cases where the magnetic field can be considered uniform in a region much larger than the region where the wave function of the atom takes on appreciable values; in these cases the eigenvectors of $\varepsilon$ coincide with the eigenvectors of $S_{z}$. In contrast, if the magnetic field does not fulfill this requirement, the Hamiltonian of the atom is not given by Equation (29) and its eigenvectors do not coincide with the eigenvectors of $S_{z}$. 
Let the initial state of the atom be $|\psi(0)\rangle=\left|+_{u}\right\rangle$. We shall find the state $|\psi(t)\rangle$ for $t>0: 1$ ) assuming the validity of OQM; and 2) assuming the validity of SPA.

\subsection{Evolution of the State Vector Assuming the Validity of OQM}

This evolution is ruled by the Schrödinger equation, which is a deterministic law. If $|\psi(0)\rangle=\left|+{ }_{u}\right\rangle$, per Equations (28), (31) and (32), the state at time $t$ will be

$$
|\psi(t)\rangle=\cos \frac{\vartheta}{2} \mathrm{e}^{-i \varphi / 2} \mathrm{e}^{-i \omega / 2}\left|+_{z}\right\rangle+\sin \frac{\vartheta}{2} \mathrm{e}^{i \varphi / 2} \mathrm{e}^{i \omega / 2}\left|-_{z}\right\rangle
$$

Let $\boldsymbol{w}(t)$ be the unitary vector defined by the angles

$$
\begin{gathered}
\Theta(t)=\vartheta \\
\Phi(t)=\varphi+\omega t
\end{gathered}
$$

The vector $\boldsymbol{w}(t)$ turns about the $O z$ axis with the Larmor frequency and spends a time

$$
T_{L}=\frac{2 \pi}{\omega}
$$

to complete a tour.

Let $S_{w}$ be the $w(t)$-component of $S$ and $\left|+_{w}\right\rangle$ its eigenvector corresponding to the eigenvalue $+\frac{\hbar}{2}$. On the basis of the eigenvectors of $S_{z}$ it can be expanded

$$
\left|+_{w}\right\rangle=\cos \frac{\vartheta}{2} \mathrm{e}^{-i \varphi / 2} \mathrm{e}^{-i \omega t / 2}\left|+_{z}\right\rangle+\sin \frac{\vartheta}{2} \mathrm{e}^{i \varphi / 2} \mathrm{e}^{i \omega t / 2}\left|-_{z}\right\rangle
$$

The state $|\psi(t)\rangle$ given by Equation (33) coincides with $\left|+_{w}\right\rangle$ ([13], p. 405). In a region where the magnetic field is uniform the atom performs a Larmor precession with frequency $\omega$. In particular, at $t=T_{L}, \boldsymbol{w}\left(T_{L}\right)=\boldsymbol{w}(0)=\boldsymbol{u}$ and $\left|\psi\left(T_{L}\right)\right\rangle=|\psi(0)\rangle=\left|{ }_{u}\right\rangle$.

According to OQM the atom remains in the Schrödinger channel and at time $T_{L}$ recovers its initial state.

\subsection{Possible Changes to the State Vector Assuming the Validity of SPA}

In SPA spontaneous processes are not necessarily ruled by a deterministic equation. If the system has the preferential set $\left\{N_{\varphi}\right\}$, it can either remain in the Schrödinger channel or jump to one of its preferential states. Thus, the same initial state $|\psi(0)\rangle$ may give rise to different states $|\psi(t)\rangle$.

Let the condition $0<\vartheta<\pi$ be satisfied. The set $\left\{\left|+_{z}\right\rangle,\left|-_{z}\right\rangle\right\}$ fulfills all the conditions required to be the preferential set of the silver atom in the state $|\psi(t)\rangle$ given by Equation (33); see Section 2.2. A straightforward calculation yields

$$
\langle\psi(t)|\varepsilon| \psi(t)\rangle=\left(\frac{\hbar \omega}{2}\right) \cos \vartheta
$$




$$
\begin{gathered}
\left\langle\psi(t)\left|\varepsilon^{2}\right| \psi(t)\right\rangle=\left(\frac{\hbar \omega}{2}\right)^{2} \\
\Delta \varepsilon=\left(\frac{\hbar \omega}{2}\right) \sin \vartheta \\
\frac{1}{\tau}=\omega \sin \vartheta
\end{gathered}
$$

As $\tau$ is constant, in the time interval $(0, t)$ the atom can either remain in the Schrödinger channel with probability

$$
P_{S}(t)=\mathrm{e}^{-t / \tau}=\mathrm{e}^{-\omega t \sin \vartheta}
$$

or decay to one of its preferential states $\left(\left|+_{z}\right\rangle\right.$ and $\left.\left|-_{z}\right\rangle\right)$. If it remains in the Schrödinger channel, at $t=T_{L}$ it will recover its initial state $\left|+_{u}\right\rangle$. The corresponding probability is

$$
P_{S}\left(T_{L}\right)=\mathrm{e}^{-T_{L} / \tau}=\mathrm{e}^{-\omega T_{L} \sin \vartheta}=\mathrm{e}^{-2 \pi \sin \vartheta}
$$

According to SPA the probability that the atom will remain in the Schrödinger channel is a decreasing exponential with relaxation time $\tau$. The probability that it will recover its initial state at time $T_{L}$ decreases when the polar angle $\vartheta$ increases.

\subsection{Testing OQM vs. SPA Experimental Predictions}

In the case of a silver atom in a uniform magnetic field, the experimental predictions of SPA are radically different from those yielded by OQM. This contradiction could be decided by means of the experiment described in (Figure 2).

1) Atoms leaving the oven $O$ are collimated and move along the $y$-axis.

2) They are prepared by the filter $F$ in the eigenstate $\left|{ }_{u}\right\rangle$ of $S_{u}$ given by Equation (28) with $\varphi=0$

$$
\left|+_{u}\right\rangle=\cos \frac{\vartheta}{2}\left|+_{z}\right\rangle+\sin \frac{\vartheta}{2}\left|-{ }_{z}\right\rangle
$$

Atoms in the state $\left|{ }_{u}\right\rangle$ are stopped in $F$; for details about how this filter works see ([14], pp. 16-17) [18] [37]. We assume, in addition, that at the exit of $F$ the atoms travel with velocity $v$.

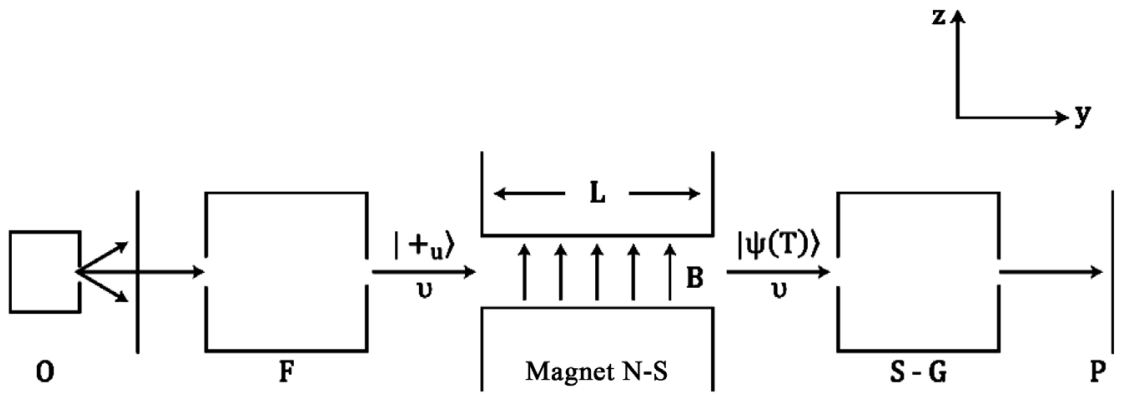

Figure 2. Schematic representation of the eperimental set-up. See text. 
3) Atoms so prepared fly through a magnet $N-S$ of length $L$ which generates a uniform magnetic field $\boldsymbol{B}$ pointing along the $z$-axis. Atoms entering one end of the magnet in the state $|\psi(0)\rangle=\left|{ }_{u}\right\rangle$ exit the other end in the state $|\psi(T)\rangle$ where

$$
T=\frac{L}{v}
$$

is the time an atom spends travelling through the magnet. The velocity of the atoms does not change during their passage through the magnet. By contrast, their spin states change, either as a result of Larmor precession or due to projection to one of their preferential states.

4) A Stern-Gerlach apparatus measures the $u$-component $S_{u}$ of the spin $S$ of the atoms in the state $|\psi(T)\rangle$.

5) The impacts of arriving atoms at plate $P$ are recorded.

We shall choose $L, v$ and $\omega$ in such a way that the time $T_{L}$ required to complete a precession tour equals the time $T$ spent by the atom in the magnet. In other words, we shall impose the condition

$$
\frac{L}{v}=\frac{2 \pi}{\omega}
$$

At the entrance as well as at the end of the magnet the magnetic field is strongly non uniform. Several authors have assumed that the evolution of a spin in a strongly non uniform magnetic field is ruled by the Schrödinger equation [37] ([38], pp. 593-598). According to SPA this is so if 1) either the atom does not have a preferential set; or 2) the atom has a preferential set, but the time it spends in such a region is so short that it "has no time" to be projected. We shall assume one of these two conditions fulfilled in the experiment just described.

\subsubsection{If $\mathrm{OQM}$ Is Valid}

The atom enters the magnet in the state $|\psi(0)\rangle=\left|+{ }_{u}\right\rangle$. While flying through the magnet it precesses around the $z$-axis with the Larmor frequency $\omega$, "it never aligns itself with the $z$-axis" ([14], p. 164). It will remain in the Schrödinger channel and at time $T$ it will recover its initial state $\left|+_{u}\right\rangle$. Therefore, a measurement of $S_{u}$ yields the result $+\frac{\hbar}{2}$ with certitude whatever the angle $\vartheta$ may be (Figures 3(a)-(c)).

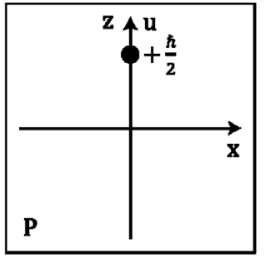

(a)

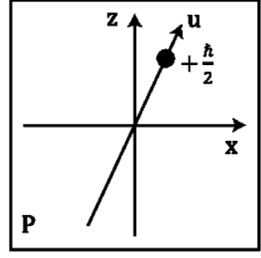

(b)

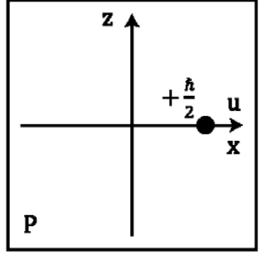

(c)

Figure 3. Registers on plate P of the measurement of $S_{u}$ according to OQM. The result is $+\frac{\hbar}{2}$ with certitude whatever the polar angle may be: (a) $\vartheta \simeq 0$; (b) $\vartheta=\pi / 6$; (c) $\vartheta=\pi / 2$. 


\subsubsection{If SPA Is Valid}

We assume, as above, that the atom enters the magnet in the state $|\psi(0)\rangle=\left|{ }_{u}\right\rangle$. When it flies through the magnet there are three possibilities:

1) It remains in the Schrödinger channel and at time $T$ it recovers its initial state. Therefore $|\psi(T)\rangle=\left|{ }_{u}\right\rangle$.

2) It jumps to the preferential state $\left|+_{z}\right\rangle$ and remains in this state until it leaves the magnet. Therefore $|\psi(T)\rangle=\left|+_{z}\right\rangle$.

3) It jumps to the preferential state $\left|-_{z}\right\rangle$ and remains in this state until it leaves the magnet. Therefore $|\psi(T)\rangle=\left|-_{z}\right\rangle$.

The corresponding probabilities will be respectively denoted $P_{S}(T), P_{+z}(T)$ and $P_{-z}(T)$. The relation

$$
P_{S}(T)+P_{+z}(T)+P_{-z}(T)=1
$$

is fulfilled.

A measurement of $S_{u}$ performed on the atom in the state $|\psi(T)\rangle=\left|+{ }_{u}\right\rangle$ yields the result $S_{u}=+\frac{\hbar}{2}$ with certitude. By contrast, if the atom is in the state $|\psi(T)\rangle=\left|+_{z}\right\rangle$, the measurement can yield either the result $S_{u}=+\frac{\hbar}{2}$ or the result $S_{u}=-\frac{\hbar}{2}$. The same applies for an atom in the state $|\psi(T)\rangle=\left|-_{z}\right\rangle$.

Let $\eta_{+z}\left(+\frac{\hbar}{2}\right)\left[\eta_{-z}\left(+\frac{\hbar}{2}\right)\right]$ be the probability that the measurement yields the result $S_{u}=+\frac{\hbar}{2}$ if the state of the atom were $\left|+_{z}\right\rangle\left(\left|-_{z}\right\rangle\right)$. The probability that a measurement of $S_{u}$ yields the result $+\frac{\hbar}{2}$ whatever the state $|\psi(T)\rangle$ be is

$$
\wp\left(+\frac{\hbar}{2}\right)=P_{S}(T)+P_{+z}(T) \eta_{+z}\left(+\frac{\hbar}{2}\right)+P_{-z}(T) \eta_{-z}\left(+\frac{\hbar}{2}\right)
$$

and the probability that a measurement of $S_{u}$ yields the result $-\frac{\hbar}{2}$ whatever the state $|\psi(T)\rangle$ be is

$$
\wp\left(-\frac{\hbar}{2}\right)=P_{+z}(T) \eta_{+z}\left(-\frac{\hbar}{2}\right)+P_{-z}(T) \eta_{-z}\left(-\frac{\hbar}{2}\right)
$$

with obvious notation. The relation

$$
\wp\left(+\frac{\hbar}{2}\right)+\wp\left(-\frac{\hbar}{2}\right)=1
$$

holds.

The following diagram illustrates the process just described.

$$
|\psi(0)\rangle=\left|+{ }_{u}\right\rangle \underset{\text { The atom goes through the magnet }}{\longrightarrow}|\psi(\mathrm{T})\rangle \underset{S_{u} \text { is measured }}{\longrightarrow}+\hbar / 2 \text { or }-\hbar / 2
$$

The probabilities $\wp\left(+\frac{\hbar}{2}\right)$ and $\wp\left(-\frac{\hbar}{2}\right)$ depend on the polar angle $\vartheta$. If 
$\vartheta \simeq 0$, the same holds for the inverse of the relaxation time $\tau$; see Equation (41). For $T \ll \tau$, the atom "has no time" to decay to one of its preferential states during its passage through the magnet. The probability that it will remain in the Schrödinger channel is $P_{S}(T) \simeq 1$. Hence

$$
\begin{aligned}
& \wp\left(+\frac{\hbar}{2}\right) \simeq 1 \\
& \wp\left(-\frac{\hbar}{2}\right) \simeq 0
\end{aligned}
$$

(Figure 4(a)). In this case the difference between SPA and OQM predictions is irrelevant.

When $\vartheta$ increases, the relaxation time $\tau$ and the probability $P_{S}(T)$ decrease and the sum $P_{+z}(T)+P_{-z}(T)$ increases, see Equations (41), (43) and (47). If the experiment is performed with a beam of atoms, the number of atoms abandoning the Schrödinger channel and exiting the magnet either in the state $\left|+_{z}\right\rangle$ or in the state $\left|-_{z}\right\rangle$ increases with $\vartheta$.

A measurement of $S_{u}$ performed on an atom in the state $\left|+_{z}\right\rangle$ may yield either the result $+\frac{\hbar}{2}$ or the result $-\frac{\hbar}{2}$; the same holds for an atom in the state $\left|-{ }_{z}\right\rangle$. As the probability $\wp\left(-\frac{\hbar}{2}\right)$ increases, the probability $\wp\left(+\frac{\hbar}{2}\right)$ decreases; see Equation (50). Plate $\mathrm{P}$ registers the impacts corresponding to both results (Figure 4(b)).

If $\vartheta=\pi / 2$, the probability that the atom will remain in the Schrödinger channel during its flight through the magnet is almost null. As $P_{S}(T) \simeq 0$, the sum $P_{+z}(T)+P_{-z}(T) \simeq 1$. Taking into account Equation (44), it is easily concluded that

$$
P_{+z}(T) \simeq P_{-z}(T) \simeq \frac{1}{2}
$$

If the atoms enter the magnet in the state $|\psi(0)\rangle=\left|+_{u}\right\rangle$, almost a half of them will decay to $\left|+_{z}\right\rangle$ and the other half will decay to $\left|-_{z}\right\rangle$ while flying through the magnet. A measurement of $S_{u}$ performed on an atom in the state $\left|+_{z}\right\rangle$ may yield either the result $+\frac{\hbar}{2}$ or the result $-\frac{\hbar}{2}$ with the same probability. This assertion is also valid if the state of the atom is $\left|-_{z}\right\rangle$. As

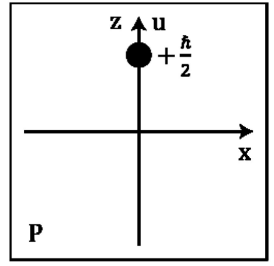

(a)

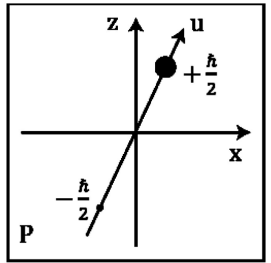

(b)

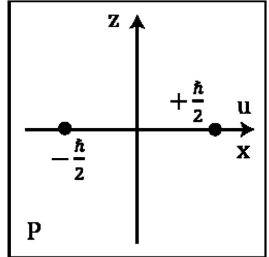

(c)

Figure 4. Registers on plate $\mathrm{P}$ of the measurement of $S_{u}$ according to SPA. The results depend on the polar angle: (a) $\vartheta \simeq 0$; (b) $\vartheta=\pi / 6$; (c) $\vartheta=\pi / 2$. 


$$
\wp\left(+\frac{\hbar}{2}\right) \simeq \wp\left(-\frac{\hbar}{2}\right) \simeq \frac{1}{2}
$$

the intensity of the traces on plate $\mathrm{P}$ corresponding to both results will be very similar (Figure 4(c)).

\subsubsection{SPA Vs. OQM: Which One Is Valid (If Any)?}

Taking into account the previous analysis we can assert that:

1) either all the atoms hit the same point on plate $P$ (which depends on the polar angle $\vartheta$ ), as shown in Figure 3; or

2) the atoms make impact in one of the two opposite points on plate $P$ as shown in Figure 4; here the positions of the opposite points depend on the polar angle and the rate between the number of impacts corresponding to the eigenvalues $-\frac{\hbar}{2}$ and $+\frac{\hbar}{2}$ of $S_{u}$ grows with $\vartheta$.

In the first case we should conclude that when flying through the magnet $N-S$ no atoms abandon the Schrödinger channel. The predictions of $O Q M$ would be confirmed. In the second case we should conclude that when flying through the magnet $N-S$ some atoms do abandon the Schrödinger channel, the number of them exiting the magnet $N-S$ increases with the polar angle and takes a maximum for $\vartheta=\pi / 2$. The predictions of SPA would be confirmed.

\section{Discusion and Conclusions}

John Bell points out: "In the beginning natural philosophers tried to understand the world around them. Trying to do that they hit upon the great idea of contriving artificially simple situations in which the number of factors involved is reduced to a minimum. Divide and conquer. Experimental science was born. But experiment is a tool. The aim remains: to understand the world. To restrict quantum mechanics to be exclusively about piddling laboratory operations is to betray the great enterprise. A serious formulation will not exclude the big world outside the laboratory" [20].

OQM is fine for all practical purposes. But besides excluding the big world outside the laboratory, its formulation lacks precision. The central notions of system, apparatus and measurement are neither included as primitive concepts nor defined in the theory. To circumvent this ambiguity-says Bell-discretion and good taste (born from experience) are needed ([39], p. 160). It would be perhaps possible to improve OQM by replacing these ambiguous notions. "However, the idea that quantum mechanics, our most fundamental physical theory, is exclusively even about the results of experiments would remain disappointing" [20].

SPA is a version of quantum theory grounded on philosophical realism. It is not about the results of experiments; it talks about what happens [16]. It has been precisely formulated [12]. The notion of system is introduced as a primitive concept and those of apparatus and measurement are not included in its formulation. Spontaneous and measurement processes are treated on the same 
footing [16]. In SPA transitions to the continuum are spontaneous processes. The expressions of the probability density per unit interval of energy resulting from SPA and from OQM treatments coincide approximately and Fermi's golden rule is obtained [15].

In the framework of OQM, it is agreed that time-dependent perturbation theory (TDPT) must be used for solving all problems involving time, including spontaneous time-dependent processes. We have questioned TDPT on the following grounds: accounting for spontaneous time-dependent processes requires the application of a law (the projection postulate) which is not valid in such processes [40]. In the same direction, contradictions reminiscent of Zeno's paradoxes of motion have been pointed out [41]. By contrast, SPA has no relation with TDPT. Hence it does not confront these issues.

According to SPA the Schrödinger equation not only rules processes where the system has no preferential set, but it also rules most processes where the system does have a preferential set. Spontaneous projections seldom occur. It is worth noting that spontaneous projections and projections resulting from OQM measurements share several traits. In particular, the mathematical expressions for the corresponding probabilities are quite similar; see Section 2.3.

The rule forcing the state vector to remain continuous as long as no measurements are performed poses a serious problem for OQM. By contrast, assuming that spontaneous projections are natural processes, SPA succeeds in stopping the endless ramifications resulting from Schrödinger evolution; see Section 2.4. Hence if SPA is valid there should be no paradoxes such as that of Schrödinger's cat.

SPA exhibits several advantages over OQM, but OQM enjoys the prestige of old age. In order to put both theories to the test, in this paper we suggest an experiment where at least one of them should fail. We study the behavior of a silver atom, initially in an eigenstate of the $u$-component of the spin, placed in a uniform magnetic field.

Assuming the validity of OQM, in Section 3.1 we find the state of the atom at a certain time $T_{L}$.

Assuming the validity of SPA, in Section 3.2 we find the possible states of the atom at the same time $T_{L}$.

In this particular case, the experimental predictions of SPA are radically different from those yielded by OQM. The resulting contradiction could be decided by means of the experiment described in Section 3.3.

Until today OQM experimental predictions seem to have no exceptions. Should the experiment mentioned yield the results predicted by SPA, it would be the first time that OQM experimental predictions fail.

\section{Acknowledgements}

We are grateful to Professors D.R. Bes, J.C. Centeno, F.G. Criscuolo, C.A. Fernández Bareilles and F. Laloë for fruitful discussions. We are indebted to Professor D. A. Morales for a critical review of the manuscript. We thank 
Mariana Delbue for her help with stylistic matters and Carlos Valero for his assistance with the figures and the transcription of the manuscript into LaTex.

\section{Conflicts of Interest}

The author declares no conflicts of interest regarding the publication of this paper.

\section{References}

[1] Burgos, M.E. (1987) Foundations of Physics, 17, 809-812. https://doi.org/10.1007/BF00733269

[2] Einstein, A. (1931) James Clerk Maxwell: A Commemoration Volume. Cambridge University Press, Cambridge.

[3] Bunge, M. (1985) Treatise on Basic Philosophy, Vol. 7, Philosophy of Science \& Technology. D. Reidel Publishing Company, Dordrecht, Boston, Lancaster.

[4] Dirac, P.A.M. (1958) The Principles of Quantum Mechanics. Clarendon Press Oxford, Oxford. https://doi.org/10.1063/1.3062610

[5] von Neumann, J. (1932) Mathematische Grundlagen der Quantenmechanik. Springer, Berlin.

[6] Tegmar, M. and Wheeler, J. (2001) Scientific American, 284, 54-61. https://doi.org/10.1038/scientificamerican0201-68

[7] Jammer, M. (1974) The Philosophy of Quantum Mechanics. John Wiley \& Sons, New York.

[8] Peres, A. (1993) Quantum Theory: Concepts and Methods. Kluwer Academic Publishers, Dordrecht.

[9] Peres, A. (1985) Foundations of Physics, 15, 201-205. https://doi.org/10.1007/BF00735292

[10] Petersen, A. (1963) Bulletin of the Atomic Scientists, 19, 8-14. https://doi.org/10.1080/00963402.1963.11454520

[11] Burgos, M.E. (1998) Foundations of Physics, 28, 1323-1346. https://doi.org/10.1023/A:1018826910348

[12] Burgos, M.E. (2018) JMP, 9, 1697-1711. https://doi.org/10.4236/jmp.2018.98106

[13] Cohen-Tannoudji, C., Diu, B. and Laloë, F. (1977) Quantum Mechanics. John Wiley \& Sons, New York, London, Sydney, Toronto.

[14] Bes, D.R. (2004) Quantum Mechanics. Springer, Berlin. https://doi.org/10.1007/978-3-662-05384-3

[15] Burgos, M.E. (2008) Foundations of Physics, 38, 883-907. https://doi.org/10.1007/s10701-008-9213-5

[16] Burgos, M.E. (2015) The Measurement Problem in Quantum Mechanics Revisited. In: Pahlavani, M., Ed., Selected Topics in Applications of Quantum Mechanics, INTECH, Croatia, 137-173. https://doi.org/10.5772/59209

[17] Burgos, M.E. (2010) JMP, 1, 137-142. https://doi.org/10.4236/jmp.2010.12019

[18] Burgos, M.E. (2015) JMP, 6, 1663-1670. https://doi.org/10.4236/jmp.2015.611168

[19] Born, M. (1971) The Born Einstein Letters, Letters 50, 53 and 81. The Mcmillan Press Ltd., London.

[20] Bell, J.S. (1990) Physics World, 3, 33-40. https://doi.org/10.1088/2058-7058/3/8/26 
[21] Laloë, F. (2001) American Journal of Physics, 69, 655-701. https://doi.org/10.1119/1.1356698

[22] Ballentine, L.E. (1970) Reviews of Modern Physics, 42, 358-381. https://doi.org/10.1103/RevModPhys.42.358

[23] Ballentine, L.E. (1972) American Journal of Physics, 40, 1763-1771. https://doi.org/10.1119/1.1987060

[24] Bohm, D.A. (1952) Physical Review, 85, 166-179. https://doi.org/10.1103/PhysRev.85.166

[25] Bohm, D.A. (1952) Physical Review, 85, 180-193. https://doi.org/10.1103/PhysRev.85.180

[26] Everett, H. (1957) Reviews of Modern Physics, 29, 454-462. https://doi.org/10.1103/RevModPhys.29.454

[27] Wikipedia, The Free Encyclopedia: Many-Worlds Interpretation.

[28] Griffith, R.B. (1984) Journal of Statistical Physics, 36, 219-272. https://doi.org/10.1007/BF01015734

[29] Omnès, R. (1988) Journal of Statistical Physics, 53, 893-932. https://doi.org/10.1007/BF01014230

[30] Zurek, W. (1991) Physics Today, 44, 36-44. https://doi.org/10.1063/1.881293

[31] Zurek, W. (2003) Reviews of Moders Physics, 75, 715-775. https://doi.org/10.1103/RevModPhys.75.715

[32] Ghirardi, G.C., Rimini, A. and Weber, T. (1986) Physical Review D, 34, 470. https://doi.org/10.1103/PhysRevD.34.470

[33] Ghirardi, G.C., Rimini, A. and Weber, T. (1987) Foundations of Physics C, 18, 1-27. https://doi.org/10.1007/BF01882871

[34] Bell, J.S. (1984) CERN-TH, 4035, 159-166. https://doi.org/10.1142/9789812386540_0017

[35] Sudbery, A. (1984) Annals of Physics, 157, 512-536. https://doi.org/10.1016/0003-4916(84)90070-8

[36] Sudbery, A. (2002) Studies in History and Philosophy of Science. Part B: Studies in History and Philosophy of Modern Physics, 33, 387-411. https://doi.org/10.1016/S1369-8486(02)00003-1

[37] Feynman, R.P., Leighton, R.B. and Sands, M. (1963) Lectures on Physics, Vol. III. Addison-Wesley, Reading, Chapter 5.

[38] Bohm, D.A. (1951) Quantum Theory. Prentice-Hall, Princeton.

[39] Bell, M., Gottfried, K. and Veltman, M. (2001) John S. Bell on the Foundations of Quantum Mechanics. Word Scientific, Singapore. https://doi.org/10.1142/4757

[40] Burgos, M.E. (2016) JMP, 7, 1449-1454. https://doi.org/10.4236/jmp.2016.712132

[41] Burgos, M.E. (2017) JMP, 8, 1382-1397. https://doi.org/10.4236/jmp.2017.88087 